\title{
A comprehensive study of the spectral and photophysical properties of arylthiophenes
}

\author{
J. Seixas de Melo*, J. Pina, L.M. Rodrigues, R.S. Becker \\ Department of Chemistry, University of Coimbra, 3004-535 Coimbra, Portugal \\ Received 23 May 2007; received in revised form 16 July 2007; accepted 17 July 2007 \\ Available online 22 July 2007
}

\begin{abstract}
A series of conjugated oligomers with rigid (fused-ring) structure, consisting in different polycyclic aromatic hydrocarbons (PAHs) possessing a single thiophene unit, here designated as arylthiophenes, were investigated in solution. For presentation clarity, the compounds were organized in two different groups. The first group includes the compounds where one, two, three and four benzene rings are added to a thiophene unit (a compound with two dibenzothiophene units was also investigated) and the second group includes those compounds where a single thiophene ring is located at different positions in a skeletal-type polyaromatic hydrocarbon (in the present case phenanthrene). The study includes a complete spectroscopic evaluation (including singlet-singlet and triplet-triplet absorption, fluorescence and phosphorescence spectra) as well as a photophysical evaluation (fluorescence, phosphorescence and triplet lifetimes together with fluorescence and triplet occupation quantum yields. With all the above, a complete set of deactivation rate constants $\left(k_{\mathrm{F}}, k_{\mathrm{IC}}\right.$ and $\left.k_{\mathrm{ISC}}\right)$ was determined. From the fluorescence and phosphorescence spectra the energy of the lowest lying singlet and triplet states $\left(\mathrm{S}_{1}\right.$ and $\left.\mathrm{T}_{1}\right)$ and the energy of splitting between the two states $\left(\Delta E_{\mathrm{S}_{1}-\mathrm{T}_{1}}\right)$ was obtained. Semi-empirical ZINDO/S-CI calculations were performed and corroborated the nature and state order experimentally obtained.
\end{abstract}

(c) 2007 Elsevier B.V. All rights reserved.

Keywords: Photochemistry; Photophysics; Arylthiophene; Fluorescence; Phosphorescence; Quantum yields; OLEDs; Oligomer; Laser flash photolysis; Thiophene; Phenanthrene

\section{Introduction}

Since the pioneering discovery and report in 1977 [1] of Shirakawa, MacDiarmid and Heeger that organic conjugated polymers (OCPs), if appropriately doped, were found to be conductive materials. The conjugated polymers and oligomers are therefore amongst the most important advanced materials currently under investigation, with a significant number of real and potential applications. Within the most popular OCP's, we find the polyacetylene derivatives, polyfluorene and polytiophenes (thiophene based polymers) which have gained considerable interest, essentially due to their chemical stability and potential for charge transport and light emitting diode (LED) applications. More recently there has been considerable interest in new families of synthetic thiophene-derivatives in which among others we find the poly(isothianaphthenes) [2,3] and the poly(dithienothiophenes) [4] which have showed promising

\footnotetext{
* Corresponding author. Fax: +351 239827703.

E-mail address: sseixas@ci.uc.pt (J. Seixas de Melo).
}

conducting properties. A general and common feature of these new polythiophenes is that they present more structural rigidity. Following this line of structural interest, new benzothiophenes derivatives have also been considered as promising compounds for OLED applications [5-9].

Among other important issues, is the consideration of deactivation of the excited states, in particular the decay of the singlet excited state since the great majority of the emission of these polymers arises from this state. Also a subject of intense investigation is phosphorescent organic light emitting diodes (OLEDs) [10] since in general the known OLEDs have very poor emission efficiency. Phosphorescence has been observed with a longlived lifetime following photoexcitation of a frozen solution of a ladder type poly( $p$-phenylene), MeLPPP [11,12] and from thin films of polyfluorene [13,14].

For electroluminescence purposes, although different estimates exist for the ratio of singlet-to-triplet excited states produced via charge recombination (between positive and negative charge carriers) [15], it is generally accepted that triplet state formation is one of the major causes of efficiency loss in these systems. Since electroluminescence is normally only observed 
from the short-lived singlet excited state, triplet states are consequently acting as channels for decreasing the efficiency of a LED device. The introduction of emissive triplets in polymers or copolymers through coupling of known emissive fragments is consequently a valid strategy to improve the emissive response of an organic light emitting device [10].

With LEDs it is frequent to differentiate between low molecular weight (oligomeric and small molecule) and high molecular weight (polymeric) systems and there is frequently a relationship between the properties of these two groups, i.e., between the polymers and their respective oligomers [16]. In fact, one route to the understanding of the more complex polymer systems involves the study of their oligomeric derivatives. In addition to the more well-defined structure of these, they sometimes have the advantage of being more soluble in solution and therefore easier to process as thin films for solid state applications. Recently new "cruciform" oligothiophene-like systems have been considered as new and promising soluble and easily processed long chain-oligomers with increased solubility and morphologic stability [17]. Also, recently other oligomeric structures based on acenes (pentacene and anthracene) derivatives containing the thiophene ring have showed promising properties for organic semiconductor applications [18]. A recent review by J.E. Anthony clearly outlines the state of the art and the pontential applications of these acene-like compounds in the domain of organic electronics [19].

In this work, we will present a comprehensive photophysical study on aromatic-thiophene, arylthiophene oligomers containing one or two thiophene units linked to condensed aromatic hydrocarbons (with a different number of benzene units). Although the compounds display low fluorescence yields, they efficiently radiatively deactivate through the lowest triplet state which can be used to improve and tune modern organic conjugated polymers. From the six compounds here investigated, some data is available regarding some photophysical properties of the compounds presenting the simplest structures: benzothiophene [20] and dibenzothiophene [20,21]. However, even in these cases, only a limited amount of data is presented. In this work, a study is carried out involving the foregoing two compounds which are integrated with four others not previously characterized from a detailed photophysical perspective.

\section{Experimental}

\subsection{Materials}

The thianaphthene (TN) and benzo[b]naphtho[2,1-d]thiophene (BDFS) were purchased from Lancaster Synthesis, Dibenzothiophene (DBT) from Aldrich and were all purified by vacuum sublimation with a cold-finger device. The bis-dibenzothiophene (BDBT), phenanthro[9,10-b]thiophene (TBTM) and 3-thia-cyclopenta[c]phenanthrene (TBTE) were kindly supplied by Dr. Hans Wynberg (University of Groningen) and were used as received.

The solvents used were of spectroscopic or equivalent grade. The concentrations of the solutions ranged from $1 \times 10^{-5}$ to $10^{-7} \mathrm{M}$ and were deoxygenated by bubbling with $\mathrm{N}_{2}$ or $\mathrm{Ar}$.
Ethanol was dried over $\mathrm{CaO}$ and 1,4-dioxane was purified by the procedure described in Ref. [22].

\subsection{Methods}

Absorption and luminescence spectra were recorded on Shimadzu UV-2100 and Horiba-Jobin-Ivon SPEX Fluorog 3-22 spectrometers, respectively. For phosphorescence a 1934 D phosphorimeter accessory was used. Fluorescence and phosphorescence spectra were corrected for the wavelength response of the system.

The fluorescence quantum yields were measured using $\alpha$-bithiophene $\left(\phi_{\mathrm{F}}=0.014\right.$ in ethanol) [23], $\alpha$-terthienyl $\left(\phi_{\mathrm{F}}=0.054\right.$ in ethanol) [23] and 3-chloro-7-methoxy-4methylcoumarin ( $\phi_{\mathrm{F}}=0.53$ in dioxane) [24] as standards. The fluorescence quantum yields at $77 \mathrm{~K}$ were obtained by comparison with the spectrum at $293 \mathrm{~K}$ run under the same experimental conditions and the $\phi_{\mathrm{F}}$ value was obtained by assuming that $V_{293 \mathrm{~K}} / V_{77 \mathrm{~K}}=0.8[25]$.

Phosphorescence quantum yields were obtained using benzophenone as standard, $\phi_{\mathrm{Ph}}=0.84$ [25].

The molar extinction coefficients $(\varepsilon)$ were obtained from the slope of the plot of the absorption with seven solutions of different concentrations versus the concentration (correlation coefficients $\geq 0.999$ ).

Radiative lifetime $\tau_{\mathrm{R}}$ was calculated using the Strickler-Berg equation [26]; a more detailed procedure of how this was obtained (with related compounds) can be found in Refs [23,27].

Fluorescence decays were measured using a home-built TCSPC apparatus with an $\mathrm{N}_{2}$ filled IBH 5000 coaxial flashlamp as excitation source, Jobin-Ivon monochromators, Philips XP2020Q photomultiplier, and Canberra instruments TAC and MCA. Alternate measurements $(1000 \mathrm{cpc})$ of the pulse profile at 285,337 or $356 \mathrm{~nm}$ and the sample emission were performed until 1 to $2 \times 10^{4}$ counts at the maximum were reached [28]. The fluorescence decays were analysed using the modulating functions method of Striker with automatic correction for the photomultiplier "wavelength shift" [29].

Triplet absorption spectra, intersystem crossing quantum yields and triplet molar absorption coefficients were obtained using laser flash photolysis equipment and by the pulse radiolysis technique [30].

The laser flash photolysis consists of an Applied Photophysics apparatus pumped by a Nd:YAG laser (Spectra Physics) with excitation wavelength 355 or $266 \mathrm{~nm}$ [27]. The detection system is at right angles to the excitation beam and a pulsed $150 \mathrm{~W}$ Xe lamp is used to analyze the transient absorption. The signal is fed into a HP digital analyzer and transferred to an IBM RISC computer where the OD at different wavelengths and different delays after flash are collected using the appropriate software (Applied Photophysics).

First-order kinetics was observed for the decay of the lowest triplet state. The transient spectra $(300-700 \mathrm{~nm})$ were obtained by monitoring the optical density change at $5-10 \mathrm{~nm}$ intervals and averaging at least 10 decays at each wavelength. To avoid multiphoton and T-T annihilation effects, special care was taken to have optically matched dilute solutions (abs $\approx 0.2$ in a $10 \mathrm{~mm}$ 
square cell) and low laser energy $(\leq 2 \mathrm{~mJ})$ in determining the triplet yields.

The pulse radiolysis experiments were obtained using the Free Radical Research Facility, Daresbury, UK. Here, 200 ns to $2 \mu \mathrm{s}$ high energy electron pulses from a $12 \mathrm{MeV}$ linear accelerator were passed through solutions in a $2.5 \mathrm{~cm}$ optical pathlength quartz cuvette attached to a flow system, as has been described in detail elsewhere [31]. All solutions were bubbled with argon for about $30 \mathrm{~min}$ before experiments.

The triplet molar absorption coefficients were obtained by the energy transfer method. The triplet state molar absorption coefficients were determined using biphenyl, $\varepsilon_{\mathrm{T}}=27100 \mathrm{M}^{-1} \mathrm{~cm}^{-1}$ $(360 \mathrm{~nm})$ as triplet energy donors [25]. The concentrations for the compounds studied were $10^{-5} \mathrm{M}$ and they were dissolved in $10 \mathrm{mM}$ benzene solutions of biphenyl. Before experiments, all solutions were degassed with argon for $\approx 30 \mathrm{~min}$ and sealed [32]. The molar triplet-triplet molar absorption coefficients were then determined at the analytical wavelength for triplet quantum yield determinations from Eq. (1): [33]

$\frac{\varepsilon_{\mathrm{TT}}^{\mathrm{D}}}{\varepsilon_{\mathrm{TT}}^{\mathrm{A}}}=\frac{\Delta \mathrm{OD}^{\mathrm{D}}}{\Delta \mathrm{OD}^{\mathrm{A}}}$

where $\varepsilon_{\mathrm{TT}}^{\mathrm{D}}$ and $\varepsilon_{\mathrm{TT}}^{\mathrm{A}}$ are the triplet state molar absorption coefficients of donor and acceptor respectively; $\triangle \mathrm{OD}^{\mathrm{D}}$ is the maximum absorbance from the transient triplet-triplet absorption spectra of the donor in the absence of acceptor; $\Delta \mathrm{OD}^{\mathrm{A}}$ is the maximum absorbance of the acceptor triplet when both the donor and acceptor are present.

The intersystem crossing yields for the compounds $\left(\phi_{\mathrm{T}}^{\mathrm{cp}}\right)$ were obtained by comparing the $\triangle \mathrm{OD}$ at $415 \mathrm{~nm}$ of ethanol solutions (optically matched at the laser excitation wavelength) of naphthalene (Naph, standard) and of the compound (cp) using the equation: [27,34]

$\phi_{\mathrm{T}}^{\mathrm{cp}}=\frac{\varepsilon_{\mathrm{TT}}^{\mathrm{Naph}}}{\varepsilon_{\mathrm{TT}}^{\mathrm{cp}}} \cdot \frac{\Delta O \mathrm{OD}_{\mathrm{max}}^{\mathrm{cp}}}{\Delta \mathrm{OD}_{\max }^{\mathrm{Naph}}} \cdot \phi_{\mathrm{T}}^{\mathrm{Naph}}$

Room-temperature singlet oxygen phosphorescence was detected at $1270 \mathrm{~nm}$ using a Hamamatsu R5509-42 photomultiplier, cooled to $193 \mathrm{~K}$ in a liquid nitrogen chamber (Products for Research model PC176TSCE-005), following laser excitation of aerated solutions at $355 \mathrm{~nm}$ (OD@355 nm=0.20), with an adapted Applied Photophysics flash kinetic spectrometer. The modification of the spectrometer involved the interposition of a Schott RG665 filter. A 600-line diffraction grating was used instead of the standard spectrometer one to extend spectral response to the infrared. The filter employed is essential to eliminate all the first harmonic contributions from the sensitizer emission in the $500-800 \mathrm{~nm}$ region from the infrared signal. Biphenyl in cyclohexane $\left(\phi_{\Delta}=0.73\right)$ was used as standard [35].

Quantum mechanical-molecular orbital calculations were performed with a procedure elsewhere reported [27]. Alternatively, we have used the Hyperchem v. 6.01. In this case, the procedure consisted of a molecular mechanics $\left(\mathrm{MM}^{+}\right)$geometry optimization followed by a second minimization with the semi-empirical PM3. The optimization of the geometries were done until a minimum of difference between consecu- tive minimization cycles of $0.0001 /(\AA$ mol $)$ was obtained. The transition energies and oscillator strengths were obtained by means of the Zerner Intermediate Neglect of Differential Overlap/Spectroscopy with Configuration Interaction (ZINDO/S-CI) technique [36]. In this level of calculation the configuration interactions (CI) extended to 10 occupied and 10 unoccupied molecular orbitals, where only the singly excited configurations were allowed, leading to 201 singly excited configurations. For the ZINDO method, (what is this letter) overlap weighting factors were 1.267 for sigma-sigma orbitals and 0.585 for pi-pi orbitals [37].

The experimental energies for the $\mathrm{S}_{1} \leftarrow \mathrm{S}_{0}$ and $\mathrm{S}_{2} \leftarrow \mathrm{S}_{0}$ transitions were taken from the lowest (energetically) vibronic bands of the two transitions. The good vibronic resolution found for the two electronic bands gives good evidence for the reasonability of this strategy for obtaining the zero energy levels of the $S_{1}$ and $\mathrm{S}_{2}$ states.

\section{Results}

The structural formulas and acronyms of the title compounds are depicted in Scheme 1. The acronyms used and the IUPAC designations of them are as follows: benzo $[b]$ thiophene or thianaphthene $(\mathrm{TN})$, dibenzothiophene (DBT), bis-dibenzothiophene (BDBT), benzo[b]naphtho[2,1d] thiophene (BDFS), phenanthro[9,10- $b]$ thiophene (TBTM) and 3-thia-cyclopenta[c]phenanthrene (TBTE).

From the above structure design, a division in two different groups was made and is based on (I) the number of phenyl rings added to a thiophene ring (group I) with the additional BDBT which also presents the effect of two DBT units in opposite positions and (II) the relative position of the thiophene ring in a phenanthrene-based skeleton (group II), see Scheme 1.

In Fig. 1 the absorption spectra of the studied molecules are depicted. From Fig. 1 it can be observed that, for the molecules belonging to group I, the absorption bands (corresponding to three lowest electronic transitions) progressively move to longer wavelength (red shift) with the number of benzene rings, i.e., with the "size" of the molecule. Based on Platt's nomenclature for the lowest singlet excited states in cata-condensed hydrocarbons $[38,39]\left({ }^{1} \mathrm{~L}_{\mathrm{a}}\right.$ for allowed transitions and ${ }^{1} \mathrm{~L}_{\mathrm{b}}$ for forbidden transitions) a parallelism can be established for the compounds studied (see below in the discussion section).

The fluorescence spectra of the compounds are depicted in Fig. 2. Again a red-shift of the emission bands is observed with the progressive addition of benzene rings, with BDBT presenting the longest wavelength emission maxima.

Fig. 3 presents the phosphorescence and the transient triplet-triplet absorption spectra. The triplet molar extinction coefficients were obtained by pulse radiolysis of benzene solutions containing biphenyl that can accept the triplet energy from benzene, and then transfer them to the arylthiophenes. Details of the procedure used can be found in Refs. [30,40] and references therein.

Some main features including the location of the absorptions (singlet-singlet and triplet-triplet) and emissions (fluorescence and phosphorescence) extinction coefficients and photophysi- 


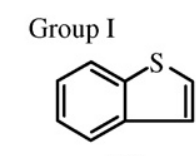

$\mathrm{TN}$<smiles>c1ccc2c(c1)sc1ccccc12</smiles>

DBT<smiles>c1ccc2c(c1)sc1cc3cc4c(cc3cc12)sc1ccccc14</smiles>

BDBT

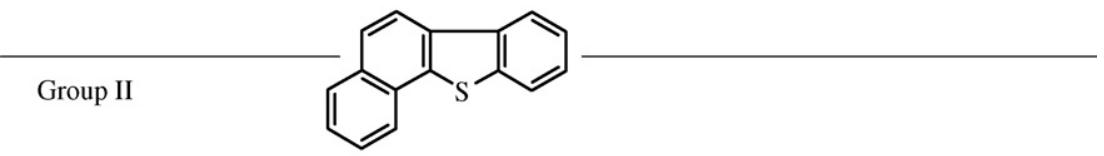

BDFS<smiles></smiles><smiles>c1ccc2c(c1)ccc1ccc3sccc3c12</smiles>

TBTE

Scheme 1.

cal data (quantum yields and rate constants) are summarized in Tables 1 and 2 .

Singlet oxygen has been detected by its characteristic phosphorescence at $1270 \mathrm{~nm}$ following photolysis of aerated solutions of the arylthiophenes. The yield of singlet oxygen formation was determined by measuring the initial phosphorescence intensity at $1270 \mathrm{~nm}$ as a function of laser intensity, and comparing the slope with that for biphenyl in cyclohexane
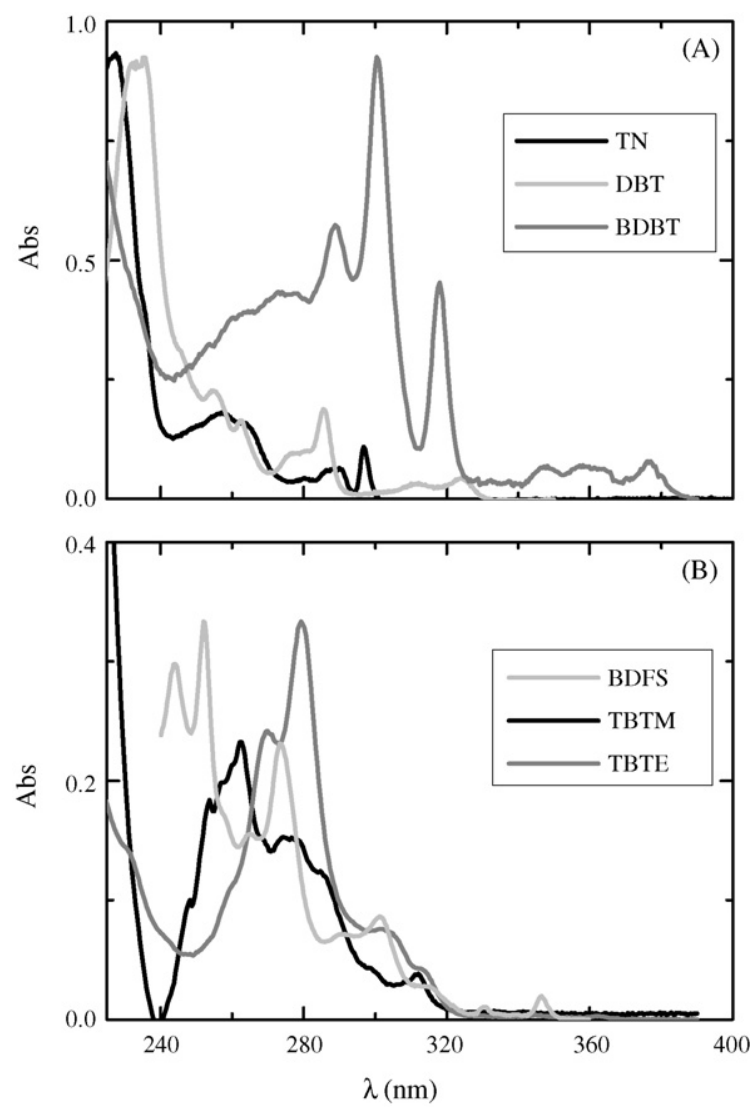

Fig. 1. Absorption spectra for the studied arylthiophenes in (A) ethanol and (B) 1,4-dioxane. as standard. From Table 2 it can be seen that the values for $\phi_{\Delta}$ and $\phi_{\mathrm{T}}$ are quite similar for group II compounds indicating that these compounds sensitize singlet oxygen formation with near unit efficiency. However for group I compounds, and with the exception of BDBT, this efficiency is decreased to $\approx 50 \%$.

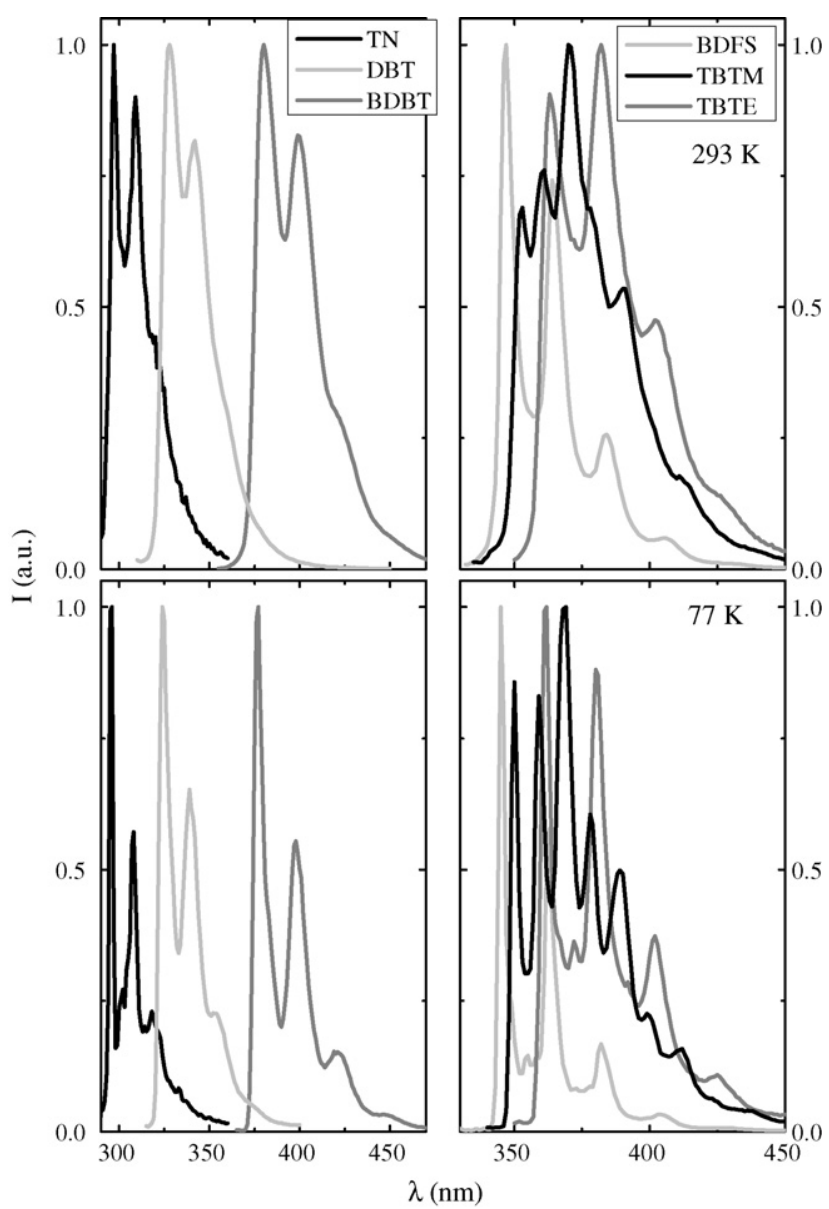

Fig. 2. Fluorescence emission spectra for the arylthiophenes in ethanol at room temperature $(293 \mathrm{~K})$ and low temperature $(77 \mathrm{~K})$. 

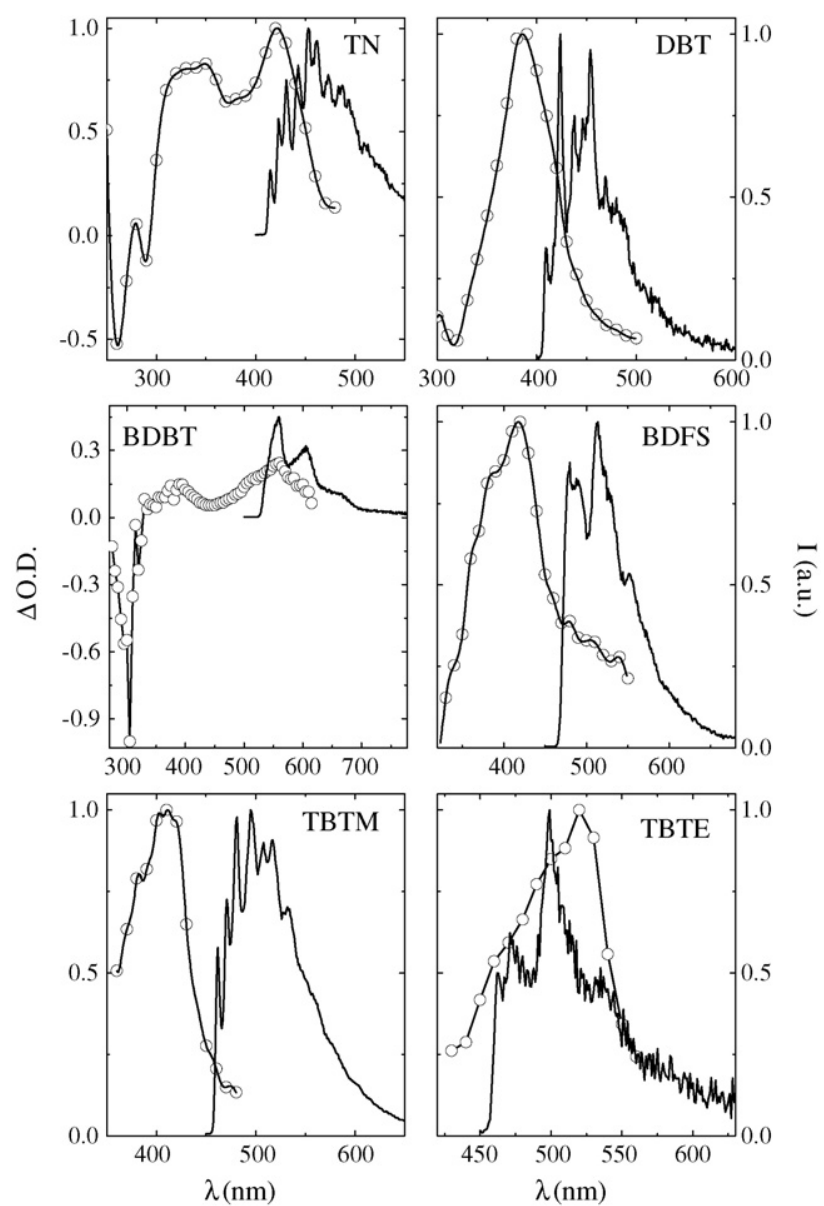

Fig. 3. Transient triplet-triplet absorption spectra (open circles) and phosphorescence emission spectra (full line) for the arylthiophenes in ethanol, $T=293 \mathrm{~K}$.

\section{Discussion}

Beginning with Group II compounds, and based on the low extinction coefficient values displayed, the lowest transition for compounds TBTE and TBTM is of ${ }^{1} \mathrm{~L}_{\mathrm{b}}$ origin in agreement with the predicted origin of this same band/state for phenanthrene [39]. However, for BDFS, the borderline compound possessing a thiophene ring instead of benzene in the middle of the phenanthrene ring, the lowest lying $\mathrm{S}_{1}$ state is now the ${ }^{1} \mathrm{~L}_{\mathrm{a}}$ which is assigned on the basis of the, low molar extinction coefficient values and radiative rate constants, see Table 1.

In the case of group I compounds, the addition of benzene rings induces a decrease in the radiative and radiationless rate constants. A recent work reports some spectral and photophysical data on compounds TN and DBT.[20] Comparison between the data in Ref. [20] and the present data reveal good agreement. However, in the data reported in Ref. [20] no $\mathrm{S}_{1} \sim \sim \mathrm{T}_{1}$ intersystem crossing yields were given and the conclusion was that the systems have a strong competition between the two $\left(\mathrm{S}_{1} \sim \sim \mathrm{S}_{0}\right.$ and $\left.\mathrm{S}_{1} \sim \sim \mathrm{T}_{1}\right)$ radiationless deactivation processes. In the present work utilizing the separation of the non-radiative constants, it is possible to observe that the intersystem crossing rate constant, $k_{\mathrm{ISC}}$, is dominating the photophysics of these compounds.

With BDBT, no significant changes in the $\phi_{\mathrm{F}}, \tau_{\mathrm{F}}$ and $k_{\mathrm{F}}$ values are seen when comparison is made with the single-unit compound DBT. However, now the $\mathrm{S}_{1} \sim \sim \mathrm{S}_{0}$ internal conversion deactivation channel becomes operative (inoperative with DBT) which leads to high $\phi_{\text {IC }}$ for BDBT. This increase in $\phi_{\text {IC }}$ (from DBT to BDBT) is made at the expenses of a decrease of the triplet yield (which decreases from 0.97 in BDBT to 0.66 in DBT) and also by a decrease in the phosphorescence quantum yield, $\phi_{\mathrm{Ph}}$, which also decreases from DBT to BDBT, see Table 2. This clearly shows that contrary to what occurs with TN and DBT, the internal conversion process is efficient in BDBT. This apparent difference between DBT and BDBT can be interpreted by a straightforward analysis of the radiationless transition theory equation $[41,42]$. The rate constant for the radiationless processes can be factored into an electronic $\left.\left(\left\langle\phi_{i}\left|H^{\prime}\right| \phi_{j}\right\rangle\right\rangle^{2}\right)$ and a vibrational term $\left(\left\langle\theta_{i} \mid \theta_{j}\right\rangle\right)$ according to [41]:

$k_{\mathrm{NR}} \propto\left\langle\phi_{i}\left|\mathrm{H}^{\prime}\right| \phi_{j}\right\rangle^{2} \sum_{j} \sum_{h} \rho\left\langle\theta_{i j} \mid \theta_{j h}\right\rangle$

Table 1

Spectroscopic data for TN, DBT, BDFS, TBTM, TBTE and BDBT in ethanol at room temperature $(293 \mathrm{~K})$ and low temperature $(77 \mathrm{~K})$

\begin{tabular}{|c|c|c|c|c|c|c|c|}
\hline Compound & $\begin{array}{l}\lambda_{\max }^{\mathrm{Abs}}(\mathrm{nm})^{\mathrm{a}} \\
293 \mathrm{~K}\end{array}$ & $\begin{array}{l}\varepsilon \\
\left(\mathrm{M}^{-1} \mathrm{~cm}^{-1}\right)\end{array}$ & $\begin{array}{l}\lambda_{\max }^{\text {Fluo }}(\mathrm{nm})^{\mathrm{a}} \\
293 \mathrm{~K}\end{array}$ & $\begin{array}{l}\lambda \lambda_{\max }^{\text {Fluo }}(\mathrm{nm})^{\mathrm{a}} \\
77 \mathrm{~K}\end{array}$ & $\lambda_{\max }^{\text {Phosph }}(\mathrm{nm})^{\mathrm{a}}$ & $\begin{array}{l}\lambda_{\max }^{\mathrm{T}_{1} \rightarrow \mathrm{T}_{\mathrm{n}}}(\mathrm{nm})^{\mathrm{a}} \\
293 \mathrm{~K}\end{array}$ & $\begin{array}{l}\varepsilon_{\mathrm{TT}}{ }^{\mathrm{c}} \\
\left(\mathrm{M}^{-1} \mathrm{~cm}^{-1}\right)\end{array}$ \\
\hline $\mathrm{TN}$ & $\frac{228}{297}$ & $\begin{array}{l}28,005 \\
3268\end{array}$ & $297, \underline{309}$ & $\underline{296}, 307$ & $\begin{array}{l}414, \\
453 \\
\end{array}$ & $350, \underline{420}$ & - \\
\hline DBT & $\frac{236}{324}$ & $\begin{array}{l}69,045 \\
3253\end{array}$ & $\underline{328}, 341$ & $\underline{324}, 339$ & $\begin{array}{l}\overline{409} \\
424\end{array}$ & 385 & - \\
\hline BDBT & $\frac{300}{377}$ & $\begin{array}{l}68,860^{\mathrm{b}} \\
6530^{\mathrm{b}}\end{array}$ & $\underline{379}, 400$ & $\underline{377}, 398$ & $\begin{array}{l}\overline{559} \\
606\end{array}$ & 560 & 21,800 \\
\hline BDFS & $\frac{252}{347}$ & $\begin{array}{l}59,840^{\mathrm{b}} \\
3406^{\mathrm{b}}\end{array}$ & $\underline{347}$ & $338, \underline{345}$ & $\begin{array}{l}480, \\
514\end{array}$ & 420 & 12,100 \\
\hline TBTM & $\frac{262}{352}$ & $\begin{array}{l}67,673^{\mathrm{b}} \\
268^{\mathrm{b}}\end{array}$ & $353, \underline{370}$ & $350, \underline{369}$ & $\begin{array}{l}\overline{462}, \\
495\end{array}$ & 415 & 39,200 \\
\hline TBTE & $\frac{279}{362}$ & $\begin{array}{l}63,754^{\mathrm{b}} \\
527^{\mathrm{b}}\end{array}$ & $363, \underline{382}$ & $\underline{362}, 380$ & $\begin{array}{l}\overline{463}, \\
499\end{array}$ & 525 & 14,600 \\
\hline
\end{tabular}

\footnotetext{
a The underlined values are the wavelength maxima.

b Value in 1,4-dioxane.

c Triplet molar extinction coefficient
} 
Table 2

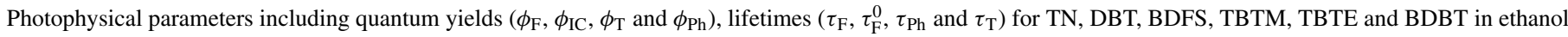
at room temperature $(293 \mathrm{~K})$ and low temperature $(77 \mathrm{~K})$

\begin{tabular}{|c|c|c|c|c|c|c|c|c|c|c|c|c|c|c|c|}
\hline Compound & $\phi_{\mathrm{F}} 293 \mathrm{~K}$ & $\phi_{\mathrm{F}} 77 \mathrm{~K}$ & $\begin{array}{l}\tau_{\mathrm{F}} \\
(\mathrm{ns})\end{array}$ & $\begin{array}{l}k_{\mathrm{F}} \\
\left(\mathrm{ns}^{-1}\right)\end{array}$ & $\begin{array}{l}k_{\mathrm{NR}} \\
\left(\mathrm{ns}^{-1}\right)\end{array}$ & $\begin{array}{l}k_{\mathrm{IC}} \\
\left(\mathrm{ns}^{-1}\right)\end{array}$ & $\begin{array}{l}k_{\mathrm{ISC}} \\
\left(\mathrm{ns}^{-1}\right)\end{array}$ & $\phi_{\text {IC }}$ & $\begin{array}{l}\tau_{\mathrm{F}}^{0} \\
(\mathrm{~ns})\end{array}$ & $\begin{array}{l}\tau_{\mathrm{R}} \\
(\mathrm{ns})\end{array}$ & $\phi_{\mathrm{Ph}}$ & $\begin{array}{l}\tau_{\mathrm{Ph}} \\
(\mathrm{s})\end{array}$ & $\phi_{\mathrm{T}}$ & $\phi_{\Delta}$ & $\begin{array}{l}\tau_{\mathrm{T}} \\
(\mu s)\end{array}$ \\
\hline $\mathrm{TN}$ & 0.019 & 0.021 & 0.28 & 0.069 & 3.50 & $\approx 0$ & 3.50 & $\approx 0$ & 15 & 31 & 0.44 & 0.31 & $0.98^{\mathrm{a}}$ & 0.45 & 3 \\
\hline DBT & 0.034 & 0.039 & 1.05 & 0.032 & 0.92 & $\approx 0$ & 0.92 & $\approx 0$ & 31 & 31 & 0.37 & 1.56 & $0.97^{\mathrm{b}}$ & 0.65 & 3 \\
\hline BDBT & 0.032 & 0.038 & 0.79 & 0.041 & 1.23 & 0.39 & 0.84 & 0.31 & 25 & 15 & 0.20 & 0.21 & 0.66 & 0.67 & 44 \\
\hline BDFS & 0.037 & 0.046 & 1.47 & 0.025 & 0.66 & 0.18 & 0.48 & 0.26 & 40 & 29 & 0.18 & 0.29 & 0.70 & 0.60 & 43 \\
\hline ТВТM & 0.019 & 0.022 & 6.65 & 0.003 & 0.15 & 0.11 & 0.041 & 0.71 & 350 & 373 & 0.20 & 0.62 & 0.27 & - & 20 \\
\hline TBTE & 0.010 & 0.009 & 2.28 & 0.004 & 0.43 & 0.14 & 0.29 & 0.32 & 228 & 190 & 0.22 & 1.46 & 0.67 & 0.71 & 29 \\
\hline
\end{tabular}

$k_{\mathrm{F}}=\frac{\phi_{\mathrm{F}}}{\tau_{\mathrm{F}}} ; k_{\mathrm{NR}}=\frac{1-\phi_{\mathrm{F}}}{\tau_{\mathrm{F}}} ; k_{\mathrm{IC}}=\frac{1-\phi_{\mathrm{F}}-\phi_{\mathrm{T}}}{\tau_{\mathrm{F}}} ; k_{\mathrm{ISC}}=\frac{\phi_{\mathrm{T}}}{\tau_{\mathrm{F}}} ; \phi_{\mathrm{IC}}=1-\phi_{\mathrm{F}}-\phi_{\mathrm{T}} ; \tau_{\mathrm{F}}^{0}=\frac{\tau_{\mathrm{F}}}{\phi_{\mathrm{F}}}$.

a Value from Ref [44].

b From Ref [45].

where $\rho$ is the density of interacting states. According to the energy gap law for radiationless transitions, the greater is the energetic difference between two interacting vibronic levels, the smaller the value the vibronic overlap factor $\left\langle\theta_{i} \mid \theta_{j}\right\rangle$ and consequently the lower will be $k_{\mathrm{NR}}$. This establishes that the $k_{\mathrm{NR}}$ increases exponentially with the decrease of the energetic difference, $E_{i}-E_{j}$, between the $\mathrm{S}_{\mathrm{i}}$ and $\mathrm{S}_{\mathrm{j}}$ states. This seems to be the case observed with BDBT relative to DBT. It is worth noting that in the case of Eq. (3) the radiationless rate constant should more accurately be considered as the internal conversion rate constant $k_{\mathrm{IC}}$. It is because of the large values of the intersystem crossing and fluorescence quantum yields, leading to $\phi_{\mathrm{T}}+\phi_{\mathrm{F}} \approx 1$, that the radiationless rate constant of interest here can be considered as the internal conversion rate constant and we note that in the case of $\mathrm{TN}$ and $\mathrm{DBT} k_{\mathrm{IC}} \approx 0$. It is also worth noting that a decrease in the $k_{\mathrm{NR}}$ values from TN to DBT (Table 2) is seen whereas in Fig. 5, see below, the $\mathrm{S}_{1}-\mathrm{S}_{0}$ energy gap (slightly) decreases from TN to DBT. This apparent non-validity of the energy-gap law for TN and DBT can at least be partially explained by fact that it is the uncertainty associated with the $k_{\mathrm{IC}}$ value that precludes a full validity of this law in the present case.

It is also worth noting that the fluorescence spectra are not affected, in shape and maxima, by temperature, see Table 1 and Fig. 2. Moreover the low temperature and room temperature absorption and emission spectra lead to insignificant StokesShift values see Figs. 1 and 2 and Table 1. This is presumably related to the structural rigidity of the studied compounds, which is basically identical at 293 and $77 \mathrm{~K}$. In fact, although a small increase in the vibronic resolution is observed, there is a minimum shift $(1-2 \mathrm{~nm})$ of the wavelength maxima with temperature. Identically, the $0-0$ vibronic band, taken from the overlap point of the normalized absorption and fluorescence emission spectrum, is practically identical at the two temperatures. The largest shift of the fluorescence wavelength maximum upon going from 293 to $77 \mathrm{~K}$ is observed with for DBT, with a value of $\approx 4 \mathrm{~nm}$. This is also valid for the $0-0$ bands obtained from absorption and fluorescence intersection of the 0 -vibronic level. Although the value $(\approx 4 \mathrm{~nm})$ is still small, this could be potentially explained by the fact that the molecule as a whole is not planar and shows a small deviation from planarity [43]. Also note that the $\phi_{\mathrm{F}}$ values at the two temperatures ( $293 \mathrm{~K}$ and $77 \mathrm{~K}$ ) are identical within the experimental error, see Table 1 .
In the case of Group II compounds it is clear that, with the exception of the border-line compound (BDFS), the radiative rate constant is one order of magnitude lower than the value found for compounds in Group I. This observation, together with the longer values obtained for the natural radiative lifetime (see $\tau_{\mathrm{F}}^{0}=\tau_{\mathrm{F}} / \phi_{\mathrm{F}}$ in Table 1) is clearly compatible with the forbidden nature of the lowest lying transition /state [42]. The nature of this transition seems to be a $\pi, \pi^{*}$ symmetry forbidden transition/state, ${ }^{1} \mathrm{~L}_{\mathrm{a}}$, since the nonbonding orbitals of the sulfur atom are likely to not be involved in the lowest lying excited states of these compounds. In fact, other solvents (besides the hydrogen bonding solvent ethanol, Table 1), such as methylcyclohexane and dioxane, have been used with the studied compounds and essentially no shift was observed in absorption. If the nature of the lowest singlet state was of $n, \pi^{*}$ origin (with involvement of the sulphur nonbonding orbitals) a blue shift of the lowest absorption band would be expected upon going from the nonpolar methylcyclohexane to the polar ethanol solvent.

From Table 2, it can be seen that the fluorescence lifetime increases in the order TN, DBT( $\approx$ BDBT $)$, BDFS, TBTE, TBTM. In all cases the fluorescence decays are single exponential indicating that no additional species or quenching (besides internal conversion and intersystem crossing) deactivation channels exist during the decay of the compounds, see Table 2 and Fig. 4.

The natural radiative lifetime resulting from the experimental data $\tau_{\mathrm{F}}^{0}\left(=\tau_{\mathrm{F}} / \phi_{\mathrm{F}}\right)$ and from the integrated lowest absorption band, $\tau_{R}$, shows nearly identical values clearly indicating that the integrated band corresponds to the observed state in emission, see Table 2. From Table 1 it can be observed that for all compounds, the balance between the radiative $\left(k_{\mathrm{F}}\right)$ and non-radiative $\left(k_{\mathrm{NR}}\right)$ rate constants is shifted towards the non-radiative processes. In the case of TN and DBT the preferred radiationless deactivation path is via the intersystem crossing $\mathrm{S}_{1} \sim \sim \mathrm{T}_{1}$ channel, see Table 1.

From Fig. 3 and Table 1 it can be observed that the compounds belonging to group II display nearly identical phosphorescence spectra (in shape and maxima). This is particularly valid for TBTE and TBTM where the $0-0$ band and wavelength maxima are identical, with the exception that TBTM displays more resolved spectra than TBTE. This is an opposite behavior to what was found with the singlet-singlet absorption, as well as with flu- 

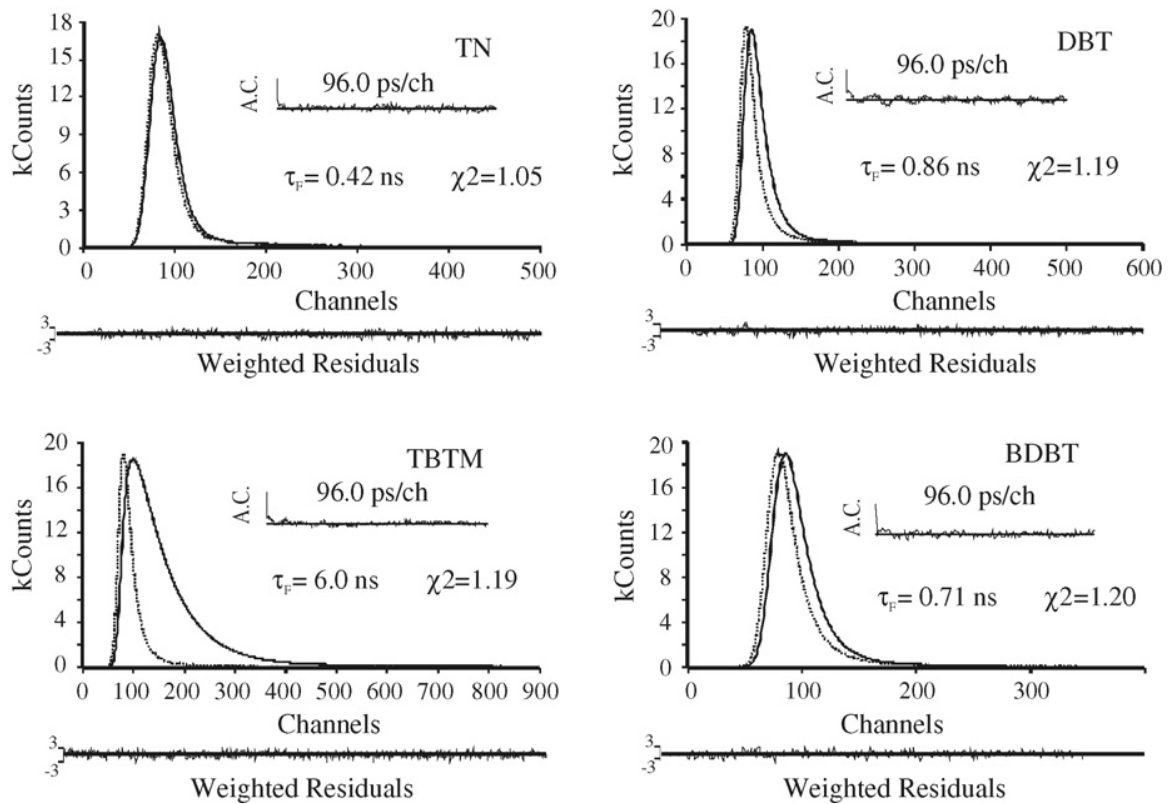

Fig. 4. Fluorescence decays for the studied compounds in ethanol. The fits are the result of adjustments to monoexpomnential decays laws. For a better judgment of the quality of the fits the autocorrelation functions (A.C.), weighted residuals and chi-square values $\left(\chi^{2}\right)$ are presented as insets. For each compound the dashed line in the decay represents the pulse instrumental response.

orescence. Contrary to what was observed in phosphorescence, the transient triplet-triplet spectra for group II compounds are different in maxima although similar in shape, see Fig. 3 and Table 1. This shows that the thiophene unit, when found in a terminal position of the phenanthrene ring, shifts the longer wavelength $T_{1} \rightarrow T_{n}$ transition and yet the relative position of the thiophene ring does not seem to affect the energy of the $0-0$ for $\mathrm{T}_{1}$.

In the case of the compounds belonging to Group I, a general increase of the wavelength maxima in the transient singlet-triplet difference spectra, $\lambda_{\max }^{\mathrm{T}_{1} \rightarrow \mathrm{T}_{\mathrm{n}}}$, with the increase in the $\pi$-electron path, i.e., with the addition of benzene or thiophene units, is observed (Table 1). The sole exception to this behavior occurs with DBT $\left(\lambda_{\max }^{T_{1} \rightarrow T_{n}}=385 \mathrm{~nm}\right.$ versus 420 for TN$)$ and is also observed in the phosphorescence spectra, where the energy for the $0-0$ in DBT is higher than for TN. A work by Glauch et al. reports a room temperature emission of DBT in a solid matrix (Whatman filter paper) with emission maximum at $450 \mathrm{~nm}$ (also present in our spectra in Fig. 3) and 0-0 at $427 \mathrm{~nm}$ which when taking into consideration the different media, is in reasonable agreement with our data [21].

In Table 3 the lowest transition energy and oscillator strength values predicted for the arylthiophenes with the ZINDO/S-CI semi-empirical method are presented. A comparison between the experimental and theoretical $S_{n} \leftarrow S_{0}$ transitions can be made by observation of Tables 1 and 3 . The procedure for geometry optimization was described in the experimental section. For all the arylthiophenes investigated, the optimized geometry was planar due to the extensive $\pi$-electron delocalization.

Although the comparison between data in Tables 1 and 3 reveals some differences in terms of absolute values, a general trend of decreasing energy gap between the $S_{1}$ and $S_{0}$ states is observed. Moreover, theory always predicts low values for the oscillator strengths of the $\mathrm{S}_{1} \leftarrow \mathrm{S}_{0}$ transition, compatible with the forbidden character assigned to the observed transitions; however, in the case of the $\mathrm{S}_{2} \leftarrow \mathrm{S}_{0}$, the values are now typically of allowed-type transitions. It is also worth noting that in the case of group II compounds the predicted values are in very close agreement with the experimental ones. One additional important question in the comparison between theory (Table 3) and experiment (Table 1) is whether the state order is correctly predicted by theory. Theory in fact does predict the lowest lying transition with low $f$ values, whereas the $S_{2} \leftarrow S_{0}$ transition displays values characteristic of allowed transitions, both in agreement with experiment.

In Fig. 5, the energies of the lowest lying singlet $\left(\mathrm{S}_{1}\right.$ and $\mathrm{S}_{2}$ ) and triplet excited states is depicted. In the case of Group I compounds, the energy value for the lowest lying singlet excited state decreases in the order TN, DBT, BDFS, BDBT, i.e., with the incremental increase in $\pi$-electron conjugation. Moreover it can be seen that with Group I compounds the $S_{1}-S_{2}$ energy gap is constant, with the exception of BDBT. However, in the case of Group II compounds this is not valid and a clear trend of decrease in the $\mathrm{S}_{1}-\mathrm{S}_{2}$ energy gap in the order BDFS, TBTM,

Table 3

Theoretical transitions and oscillator strength (for the $\mathrm{S}_{1} \leftarrow \mathrm{S}_{0}$ and $\mathrm{S}_{2} \leftarrow \mathrm{S}_{0}$ transitions) for the arylthiophenes obtained with the ZINDO/S-CI method

\begin{tabular}{llllll}
\hline Compound & $\lambda(\mathrm{nm})$ & & $f$ & \\
\cline { 2 - 3 } \cline { 5 - 6 } & $\mathrm{S}_{2} \leftarrow \mathrm{S}_{0}$ & $\mathrm{~S}_{1} \leftarrow \mathrm{S}_{0}$ & & $\mathrm{~S}_{2} \leftarrow \mathrm{S}_{0}$ & $\mathrm{~S}_{1} \leftarrow \mathrm{S}_{0}$ \\
\hline TN & 220 & 288 & 0.3074 & 0.0011 \\
DBT & 267 & 293 & & 0.5698 & 0.0020 \\
BDBT & 317 & 342 & 1.0325 & 0.0058 \\
BDFS & 260 & 328 & & 1.2549 & 0.0159 \\
TBTM & 258 & 341 & & 0.2751 & 0.0035 \\
TBTE & 271 & 344 & 1.1831 & 0.0058 \\
\hline
\end{tabular}




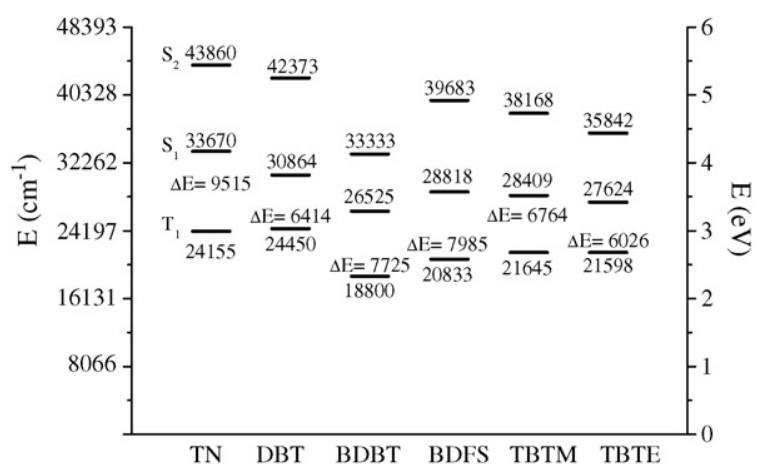

Fig. 5. Diagram with the energy values for the lowest lying singlet $\left(S_{1}\right.$ and $\mathrm{S}_{2}$ ), triplet excited states and singlet-triplet energy splitting of the studied compounds.

TBTE is found. Yet, the energy for the $S_{1}$ state is basically identical for all Group II compounds $\left(27,600-28,800 \mathrm{~cm}^{-1}\right)$, suggesting that the photophysical characteristics of the compounds result from the degree of mixing of the $S_{2}$ into $S_{1}$ [24]. With Group I compounds, the singlet to triplet energy splitting, $\Delta E_{\mathrm{S}_{1}-\mathrm{T}_{1}}$, decreases in the same order as above, with the sole exception of DBT, see Fig. 5. With all the compounds the $\Delta E_{\mathrm{S}_{1}-\mathrm{T}_{1}}$, is approximately identical $\left(\approx 6000-7000 \mathrm{~cm}^{-1}\right)$ with the exceptions of TN and BDFS with values of $8000-9500 \mathrm{~cm}^{-1}$. This pattern shows that the energy difference between $S_{1}$ and $T_{1}$ states is not ruling the efficiency of the singlet to triplet intersystem crossing. Moreover, the degree of singlet-triplet coupling does not seem to be associated with the classical heavy atom effect since basically all the compounds possess identical number of sulfur atoms. However, in the case of DBT and BDBT the comparison between the $\Delta E_{\mathrm{S}_{1}-\mathrm{T}_{1}}$ values shows that it increases for the latter. In this case, the dependence of spin-orbital operator on the $\Delta E_{\mathrm{S}_{1}-\mathrm{T}_{1}}$ [41] seems to prevail with BDBT having a higher value, $7725 \mathrm{~cm}^{-1}$ for BDBT versus $6414 \mathrm{~cm}^{-1}$ for DBT, and consequently a lower $\phi_{\mathrm{T}}$ value, 0.66 for BDBT versus 0.97 for DBT- see Table 2 and Fig. 5. The latter observation could explain the more favorable internal conversion route for the dimer BDBT $\left(\phi_{\mathrm{IC}}=0.31\right)$ relative to the monomer DBT $\left(\phi_{\mathrm{IC}} \approx 0\right)$.

The above data seem to establish that the gradual increase in the $\pi$-electron path by adding additional benzene rings does not changes the nature of the state order and the relative positions of the $S_{1}, S_{2}$ and $T_{1}$ states. As a consequence of that it seems that the benzothiophene unit dictates the relative energy positions of the $S_{1}, S_{2}$ and $T_{1}$ states. In group II compounds, the phenanthrene ring seems to determine the relative position of the lowest lying states as well as of the nature of the $\mathrm{S}_{1}$ state (of forbidden nature).

\section{Conclusions}

The photophysics of six polyaromatic hydrocarbons in which one or more thiophene ring(s) has been substituted for a benzene ring was investigated in solution at room and low temperature. Emission from the singlet and triplet states was observed for all the investigated compounds. For group I compounds, with the exception of BDBT, the single-triplet intersystem crossing is the predominant deactivation channel whereas with group II compounds there is a competition between the two radiationless deactivation channels: internal conversion among the singlets and singlet to triplet intersystem crossing. Emission from the singlet state for all compounds is a minor deactivation pathway $\left(\phi_{\mathrm{F}} \sim 10^{-2}\right)$ whereas emission from the triplet has been found to be efficient ( $\left.\phi_{\mathrm{Ph}} \sim 0.2-0.44\right)$. In group II compounds, it was found that the relative position of the thiophene leads to important differences in the photophysical properties.

\section{Acknowledgements}

Financial support from FEDER and FCT (project POCI/ QUI/58291/2004) are acknowledged. J.P. acknowledges FCT for a PhD grant (SFRH/BD/18876/2004). Pulse radiolysis experiments were carried out at the Free Radical Research Facility in the Synchrotron Radiation Department of the CLRC Daresbury Laboratory, Warrington, UK, with the support of the European Commission through the project "Dynamics of Conjugated copolymers and oligomers in solution and solid state"-Improving Human Potential- Transnational Access to Major Research Infrastructures Contract HPRI-CT-2002-00183. Drs. S. Navaratnam and R. Edge (FRRF, Daresbury Laboratory, UK) are acknowledged for their excellent technical support in the pulse radiolysis experiments.

\section{References}

[1] H. Shirakawa, E.J. Louis, A.G. Macdiarmid, C.K. Chiang, A.J. Heeger, J. Chem. Soc. Chem. Commun. (1977) 578.

[2] Y. Ikenoue, F. Wudl, A.J. Heeger, Synth. Met. 40 (1991) 1

[3] W.T. Chen, G.A. Bowmaker, J.M. Seakins, R.P. Cooney, Synth. Met. 128 (2002) 215.

[4] A. Bolognesi, M. Catellani, S. Destri, R. Zamboni, C. Taliani, J. Chem. Soc. Chem. Commun. (1988) 246.

[5] M. Venanzi, G. Bocchinfuso, A. Palleschi, A.S. Abreu, P.M.T. Ferreira, M. Queiroz, J. Photochem. Photobiol. A Chem. 170 (2005) 181

[6] J.J. Morrison, M.M. Murray, X.C. Li, A.B. Holmes, S.C. Morratti, R.H. Friend, H. Sirringhaus, Synth. Met. 102 (1999) 987.

[7] X.C. Li, H. Sirringhaus, F. Garnier, A.B. Holmes, S.C. Moratti, N. Feeder, W. Clegg, S.J. Teat, R.H. Friend, J. Am. Chem. Soc. 120 (1998) 2206.

[8] H. Sirringhaus, R.H. Friend, X.C. Li, S.C. Moratti, A.B. Holmes, N. Feeder, Appl. Phys. Lett. 71 (1997) 3871.

[9] H. Sirringhaus, R.H. Friend, C. Wang, J. Leuninger, K. Müllen, J. Mat. Chem. 9 (1999) 2095.

[10] X.J. Wang, M.R. Andersson, M.E. Thompson, O. Ingandas, Thin Solid Films 468 (2004) 226.

[11] Y.V. Romanovskii, H. Bassler, Chem. Phys. Lett. 326 (2000) 51.

[12] D. Hertel, S. Setayesh, H.G. Nothofer, U. Scherf, K. Mullen, H. Bassler, Adv. Mater. 13 (2001) 65.

[13] C. Rothe, A. Monkman, Phys. Rev. B 65 (2002) 073201.

[14] C. Rothe, R. Guentner, U. Scherf, A.P. Monkman, J. Chem. Phys. 115 (2001) 9557

[15] Y. Cao, I.D. Parker, G. Yu, C. Zhang, A.J. Heeger, Nature 397 (1999) 414.

[16] J. Gierschner, J. Cornil, H.J. Egelhaaf, Adv. Mater. 19 (2007) 173.

[17] J. Pina, J. Seixas de Melo, H.D. Burrows, A. Bilge, T. Farrell, M. Forster, U. Scherf, J. Phys. Chem. B 110 (2006) 15100.

[18] M.L. Tang, T. Okamoto, Z.N. Bao, J. Am. Chem. Soc. 128 (2006) 16002.

[19] J.E. Anthony, Chem. Rev. 106 (2006) 5028.

[20] B. Wex, B.R. Kaafarani, E.O. Danilov, D.C. Neckers, J. Phys. Chem. A $110(2006) 13754$. 
[21] A. Ghauch, J. Rima, C. Fachinger, J. Suptil, M. Martin-Bouyer, Talanta 51 (2000) 807.

[22] J. Seixas de Melo, L.M. Silva, L.G. Arnaut, R.S. Becker, J. Chem. Phys. 111 (1999) 5427.

[23] R.S. Becker, J. Seixas de Melo, A.L. Maçanita, F. Elisei, J. Phys. Chem. 100 (1996) 18683.

[24] J. Seixas de Melo, R.S. Becker, F. Elisei, A.L. Maçanita, J. Chem. Phys. 107 (1997) 6062.

[25] S. Murov, I. Charmichael, G.L. Hug, Handbook of Photochemistry, M. Dekker Inc., New York, 1993.

[26] S.J. Strickler, R.A. Berg, J. Chem. Phys. 37 (1962) 814.

[27] J. Seixas de Melo, L.M. Silva, M. Kuroda, J. Chem. Phys. 115 (2001) 5625.

[28] J. Seixas de Melo, P.F. Fernandes, J. Mol. Struct. 565/566 (2001) 69.

[29] G. Stricker, V. Subramaniam, C.A.M. Seidel, A. Volkmer, J. Phys. Chem. B 103 (1999) 8612.

[30] H.D. Burrows, J. Seixas de Melo, C. Serpa, L.G. Arnaut, A.P. Monkman, I. Hamblett, S. Navaratnam, J. Chem. Phys. 115 (2001) 9601.

[31] H.D. Burrows, J. Seixas de Melo, M. Forster, R. Guntner, U. Scherf, A.P. Monkman, S. Navaratnam, Chem. Phys. Lett. 385 (2004) 105.

[32] J. Seixas de Melo, Chem. Educator 10 (2005) 29.
[33] R.V. Bensasson, E.J. Land, T.G. Truscott, Excited States and Free Radicals in Biology and Medicine, Oxford Science Publications, Oxford, 1993.

[34] C.V. Kumar, L. Qin, P.K. Das, J. Chem. Soc. Faraday Trans. 280 (1984) 783.

[35] M. Kristiansen, R.D. Scurlock, K.K. Iu, P.R. Ogilby, J. Phys. Chem. 95 (1991) 5190.

[36] M.C. Zerner, in: K.B. Lipkowitz, D.B. Boyd (Eds.), Reviews in Computational Chemistry, VCH Publishers Inc., New York, 1991, p. 313.

[37] J. Del Bene, H.H. Jaffe, J. Chem. Phys. 48 (1968) 1807.

[38] J.R. Platt, J. Chem. Phys. 17 (1949) 484.

[39] H.B. Klevens, J.R. Platt, J. Chem. Phys. 17 (1949) 470.

[40] J.S. Seixas de Melo, C. Serpa, H.D. Burrows, L.G. Arnaut, Ang. Chem. Int. Ed. Eng. 46 (2007) 2094.

[41] R.S. Becker, Theory and Interpretation of Fluorescence and Phosphorescence, Wiley-Interscience, New York, 1969.

[42] N.J. Turro, Molecular Photochemistry, W. A. Benjamin, inc., New York, 1965.

[43] R.M. Schaffrin, J. Trotter, J. Chem. Soc. A Inorg. Phys. Theor. (1970) 1561.

[44] M. Zander, G. Kirsch, Z. Naturfors. Sect. A-J. Phys. Sci. 44 (1989) 205.

[45] J.M. Bonnier, P. Jardon, J. Chim. Phys. Chim. Biol. 67 (1970) 577. 